\title{
Ultra-low-modulus Photosensitive Polybenzoxazole Systems
}

\author{
Junichi Ishii, Hideyuki Yokotsuka, Ayumi Kato, Tatsuya Kuroda and Masatoshi Hasegawa \\ Department of Chemistry, Faculty of Science, Toho University, \\ 2-2-1 Miyama, Funabashi, Chiba 274-8510
}

\begin{abstract}
This work proposes ultra-low-modulus polybenzoxazoles (PBOs), which was derived from 2,2-bis(3-amino-4-hydroxyphenyl)hexafluoropropane (6FAP), a siloxanediamine (Si-DA), isophthaloyl chloride (IPC), and pyromellitic dianhydride (PMDA). The thermally cured PBO films achieved an ultra-low modulus $(<0.44 \mathrm{GPa})$. The PBO precursor (polyhydroxyamide, PHA) films possessed a relatively high $i$-line transmittance $\left(T_{365}>\right.$ $44 \%$ ), good tetramethylammonium hydroxide (TMAH) solubility. The PHA films dispersed diazonaphthoquinone (DNQ) allowed the formation of fine positive-tone patterns. Thus, the present ultra-low-modulus PBO systems can be promising candidates as novel buffer-coating materials in semiconductor applications.
\end{abstract}

Keywords: Photosensitive Polybenzoxazoles, Ultra-low Modulus

\section{Introduction}

Aromatic polybenzoxazoles (PBOs) have been utilized in buffer-coating films in semiconductor devices for their combined excellent properties, i.e., high glass transition temperature $\left(T_{\mathrm{g}}\right)$, high resistance to chemicals, and excellent thermal stability. A remarkable merit of applying PBO systems is their high-resolution patterning ability; a diazonaphthoquinone(DNQ)-dispersed PBO precursor film (polyhydroxyamide, PHA) can give rise to fine patterns by development with a standard organic alkaline developer, $2.38 \%$ tetramethylammonium hydroxide (TMAH) aqueous solution $[1,2]$. This results from the fact that the phenolic $\mathrm{OH}$ groups in the PHA structures possess an adequate acidity $\left(p K_{\mathrm{a}}\right)$, whereas polyimide precursors as an opponent are too soluble in a TMAH solution owing to the presence of the carboxylic acid groups with a much higher acidity.

The progress of recent semiconductor technologies requires the use of thinner silicon wafers for LSI chip fabrications. Such trend causes a forthcoming serious problem, i.e., wafer deformation arising from mismatching of the linear coefficient of thermal expansion in the film plane direction (CTE) between silicon wafers (CTE $\sim 3$ ppm K$\left.{ }^{-1}\right)$ and buffer coat films $\left(50-80 \mathrm{ppm} \mathrm{K}^{-1}\right)$ as protection coatings. Two approaches to solve the serious problem are known: the use of low-CTE ( $<$ $10 \mathrm{ppm} \mathrm{K}^{-1}$ ) or low-modulus materials.

There are some attempts addressing to the development of film-processable PBO systems with considerably linear/stiff main chain structures $[3,4]$. The most important structural factor for obtaining low-CTE of PBO films is the main chain linearity/stiffness in the $\mathrm{PBO}$ structure. For example, a PBO film prepared with great difficult, which was derived from terephthaloyl chloride (TPC) and bis(3-hydroxy-4-amino)biphenyl ( $p$-HAB) derivative, showed an extremely low CTE (5.3 ppm K ${ }^{-1}$ ) [3]. However, the TPC/p-HAB system possesses a serious disadvantage in the film preparation process; once the as-polymerized PHA solution was poured into the excess of water to remove some residual polymerization reagents and unnecessary by-products, the precipitate formed becomes quite insoluble in any solvents. The use of a $p$-HAB isomer, bis(4-hydroxy-3-amino)biphenyl $(m-\mathrm{HAB})$ drastically improved the solubility at the stage of the PHA owing to its highly distorted chain structure [3]. This system also led to a high-quality of low-CTE PBO film on the conventional solution casting method and successive heating for cyclodehydration. However, this PHA film was highly colored. The poor light 
transmittance of the PHA film itself is supposed to inhibit the photoreaction of DNQ dispersed in the film. Recently, GBL, which has lower dissolution ability than NMP, is mainly used as a solvent for buffer-coating materials. Therefore, PHAs need to be highly soluble in GBL rather than NMP. However, the molecular designs toward low-CTE PBOs drastically decrease the GBL solubility of the rigid PHAs. Thus, it is very difficult to overcome the obstacles for acquiring low-CTE photosensitive PBO systems with sufficient transparency at the stage of the precursors. This situation motivated us to challenge the development of novel photosensitive PBO systems possessing sufficiently low modulus.

We have previously studied ultra-low-modulus siloxane-containing polyimides (PIs) as screen-printable cover layer materials, although it possessed no photo-patterning functionality [5]. This PI was highly soluble even in less hygroscopic solvents such as triglyme (Tri-GL) and GBL so that it provided a rather stable solution with a very high solid content ( $>60 \mathrm{wt} \%$ in GBL). However, this feature also caused a serious problem: the absence of solubility in a $2.38 \%$ TMAH solution due to its strong hydrophobicity originating from of the long siloxane chains as the flexible units.

In this work, we challenged to significantly improve the TMAH solubility of our PBO systems by a structural modification without sacrificing their inherent ultra-low modulus characteristics.

\section{Experimental Section}

\subsection{Polymerization of PHAs}

\subsubsection{Siloxane-containing PHAs}

Siloxane-containing PHAs were polymerized by a trialkyl-silylation technique [6] according to the reaction scheme shown in Fig. 1. A typical polymerization procedure using 2,2-bis(3-amino-4-hydroxyphenyl) hexafluoropropane (6FAP), siloxanediamine (Si-DA; average molecular weight $\left.=1290 \mathrm{~g} \mathrm{~mol}^{-1}\right)$, and diacid chlorides is as follows: in a $100 \mathrm{~mL}$ flask sealed with a septum cap, 6FAP $(7.0 \mathrm{mmol})$ was dissolved in $20 \mathrm{~mL}$ of dehydrated GBL containing pyridine $(28 \mathrm{mmol})$ as an $\mathrm{HCl}$ acceptor. Chlorotrimethylsilane (ClTMSi, $28 \mathrm{mmol}$ ) as a silylation reagent was slowly added to the 6FAP solution by a syringe, then stirred for $1 \mathrm{~h}$ at room temperature to quantitatively ensure silylation of the phenolic hydroxyl and the amino groups in 6FAP. After Si-DA $(3.0 \mathrm{mmol})$ and $10 \mathrm{~mL}$ of GBL were added to this solution, 4,4'-oxydibenzoyl chloride (OBC, $10.0 \mathrm{mmol}$ ) dissolved in $5 \mathrm{~mL}$ GBL was gradually added and stirred at room temperature for $1 \mathrm{~h}$. Finally, pyridine $(18 \mathrm{mmol})$ was added, and the reaction mixture was diluted with $5 \mathrm{~mL}$ GBL, then stirred at room temperature for $24 \mathrm{~h}$. A viscus light-yellowish solution of silylated PHA was formed. It was poured into a large amount of an $\mathrm{HCl}$ aqueous solution (ca. $\mathrm{pH} 4$ ) to remove the HCl-pyridine salt and to ensure desilylation of the silylated PHA. The fibrous white precipitate obtained was collected by filtration, repeatedly washed with a demineralized water and dried in vacuum at $60-100{ }^{\circ} \mathrm{C}$ for $12 \mathrm{~h}$. Isophthaloyl chloride (IPC) was also used instead of OBC. The corresponding PHAs were polymerized in a similar manner.

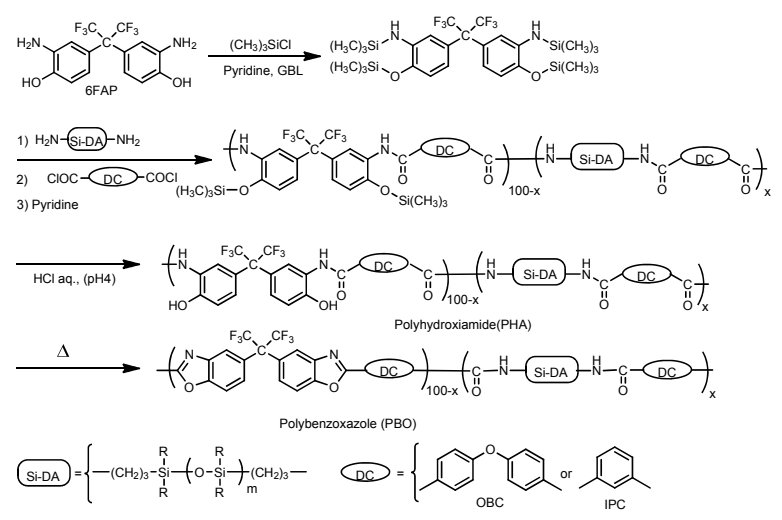

Fig. 1 Polycondensation and cyclodehydration reaction schemes for siloxane-containing PHAs.

\subsubsection{Siloxane-containing PHAs modified with PMDA}

Siloxane-containing PHAs were modified by using PMDA as a comonomer. The polymerization scheme is shown in Fig.2. The PHA was prepared as follows: in a $100 \mathrm{~mL}$ flask sealed with a septum cap, 6FAP (3.0 mmol) was dissolved in $6 \mathrm{~mL} \mathrm{GBL}$ containing pyridine (12 mmol). ClTMSi (12 mmol) was slowly added to the 6FAP solution by a syringe, then stirred for $1 \mathrm{~h}$ at room temperature. After Si-DA $(2.0 \mathrm{mmol})$ and $1 \mathrm{~mL}$ GBL were added to this solution, IPC (4.0 $\mathrm{mmol})$ dissolved in $2 \mathrm{~mL}$ GBL was gradually added and stirred at room temperature for $1 \mathrm{~h}$. Finally, PMDA $(1.0 \mathrm{mmol})$ powder, pyridine $(10 \mathrm{mmol})$, and $1 \mathrm{~mL} \mathrm{GBL}$ were added, then stirred at room temperature for $24 \mathrm{~h}$. A homogeneous/viscus solution of the PMDA-modified silylated PHA was formed. It was 
poured into a large amount of an $\mathrm{HCl}$ aqueous solution (ca. pH4). The fibrous white precipitate obtained was collected by filtration, repeatedly washed with water and dried in vacuum at $70{ }^{\circ} \mathrm{C}$ for $12 \mathrm{~h}$.

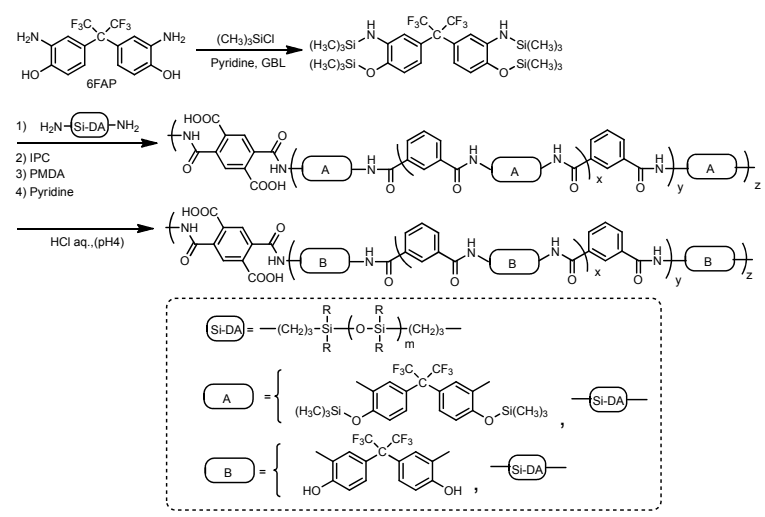

Fig. 2 Polymarization scheme for siloxane-containing PHAs modified with PMDA.

2.2. Preparation of PHA films and thermal cyclodehydration

2.2.1. Monitoring of thermal cyclization for PHA film by IR spectroscopy

The PHA powder samples were completely dissolved in GBL by stirring at room temperature for $24 \mathrm{~h}$, and the solution was coated on a silicon oxide-free $\mathrm{Si}$ wafer, and dried at $100{ }^{\circ} \mathrm{C}$ for $10 \mathrm{~min}$. The PHA films formed on the substrate were inserted in a block heater kept at established temperatures and heated for $10 \mathrm{~min}$ under a nitrogen flow. The established temperatures were increased with an interval of 20 or $30{ }^{\circ} \mathrm{C}$ until $350{ }^{\circ} \mathrm{C}$ (stepwise heating). To determine the temperature condition enough for complete cyclization, transmission-mode FT-IR spectra were measured at room temperature on a FT/IR-460 plus spectrometer (JASCO) after keeping the specimens at each temperature step for $10 \mathrm{~min}$.

\subsubsection{Preparation of $\mathrm{PBO}$ films for property evaluation}

The GBL solutions of PHAs were coated on an electrodeposited copper foil and dried at $100{ }^{\circ} \mathrm{C}$ for 10 min. The PHA films on the copper foil were heated in a vacuum-oven at $300{ }^{\circ} \mathrm{C}$ for $1 \mathrm{~h}$. The copper foil of the laminates was removed by etching it out with a $\mathrm{FeCl}_{3}$ aqueous solution. The free-standing PBO films were washed with water and dried at $100{ }^{\circ} \mathrm{C}$ for $1 \mathrm{~h}$.

\subsection{Evaluation of photosensitivity}

DNQ as a positive-type photosensitizer and an adhesion promoter were dissolved in a GBL solution of PHAs by stirring at room temperature for $24 \mathrm{~h}$ while shading to form a photosensitive film with a DNQ content of $30 \mathrm{wt} \%$. The mixed solutions were spin-coated on a silicon wafer pre-treated with a silane coupling agent, and dried at $100{ }^{\circ} \mathrm{C}$ for $10 \mathrm{~min}$. The $5-10 \mu \mathrm{m}$ thick photosensitive PHA films were irradiated at 365 $\mathrm{nm}$ by a high-pressure mercury lump (Harison-Toshiba Lighting, TOSCURE 251) with an exposure dose of $3000 \mathrm{~mJ} \mathrm{~cm} \mathrm{~cm}^{-2}$ through a photo-mask. The uv-irradiated films were developed in a $2.38 \%$ TMAH solution at room temperature, then rinsed with water at room temperature. The patterned PHA films were converted to $\mathrm{PBO}$ by heating at $300{ }^{\circ} \mathrm{C}$ for $1 \mathrm{~h}$ in vacuum. The positive-tone patterns of the PBO films were observed by a scanning electron microscope (ELIONIX, EXM-3500).

\subsection{Other measurements}

The reduced viscosities $\left(\eta_{\mathrm{red}}\right)$ of PHAs were determined at $30{ }^{\circ} \mathrm{C}$ in GBL at a concentration of $0.5 \mathrm{wt} \%$ using an Ostwald viscometer. The light transmittance at $365 \mathrm{~nm}\left(T_{365}\right)$ of PHA films was measured on an ultraviolet-visible spectrophotometer (JASCO, V-530). Mechanical properties [tensile modulus $(E)$ and elongation at break $\left(\varepsilon_{\mathrm{b}}\right)$ ] of PBO films ( $3 \mathrm{~mm}$ wide, $35 \mathrm{~mm}$ long, 15-20 $\mu \mathrm{m}$ thick) were measured using a mechanical testing machine $(\mathrm{A} \& \mathrm{D}$, Tension UTM-II) at a cross head speed of $8 \mathrm{~mm} \mathrm{~min}^{-1}$ at room temperature. Thermal stability of $\mathrm{PBO}$ films were evaluated from the $5 \%$ weight loss temperatures $\left(T_{\mathrm{d}}^{5}\right)$ at heating rate of $10{ }^{\circ} \mathrm{C} \mathrm{min}^{-1}$ in a nitrogen atmosphere by thermogravimetric analysis (TGA, NETZSCH, TG-DTA2000S). Dynamic mechanical analysis (DMA, TA Instruments Q800) was carried out at a heating rate of $5{ }^{\circ} \mathrm{C} \mathrm{min}^{-1}$ in a nitrogen atmosphere at a frequency of $0.1 \mathrm{~Hz}$ using PBO specimens $(20 \mathrm{~mm}$ long, $10 \mathrm{~mm}$ wide, and typically $20 \mu \mathrm{m}$ thick). The linear coefficients of thermal expansion (CTE) of the PBO specimen $(10 \mathrm{~mm}$ long, $5 \mathrm{~mm}$ wide, and typically $10 \mu \mathrm{m}$ thick) was measured at a heating rate of $5{ }^{\circ} \mathrm{C} \mathrm{min}{ }^{-1}$ as an average within $100-200{ }^{\circ} \mathrm{C}$ on a thermomechanical analyzer (NETZSCH, TMA 4100) with a load ( $0.5 \mathrm{~g}$ per film thickness in $\mu \mathrm{m})$ in a nitrogen flow. 


\section{Results and Discussion}

\subsection{Siloxane-containing OBC/6FAP-based PBO systems}

The siloxane-free OBC/6FAP-based homo PBO film (\#1) showed common levels of tensile modulus $(2.34 \mathrm{GPa})$ and CTE of $58 \mathrm{ppm} \mathrm{K}^{-1}$ as listed in Table 1. However, these properties can be responsible for a serious deformation problem in the silicon wafer/PBO laminates; such deformation problem is caused by thermal stress arising during the thermal cyclodehydrating reaction of PHA films. The extent of thermal stress $(\Delta \sigma)$ is expressed as a definite integral from a curing temperature $\left(T_{\text {cure }}\right)$ to room temperature $\left(T_{\mathrm{rt}}\right)$ as Eq.(1):

$$
\Delta \sigma=-\int_{T_{\text {cure }}}^{T_{\mathrm{rt}}} \frac{E_{\text {buff }}}{1-v_{\text {buff }}}\left(\alpha_{\mathrm{s}}-\alpha_{\text {buff }}\right) d T
$$

where $E_{\text {buff }}$ and $v_{\text {buff }}$ are Young's modulus and Poisson's ratio for the buffer coat materials, respectively. $\alpha_{\mathrm{s}}$ and $\alpha_{\text {buff }}$ are the in-plane CTE values of the wafer as a substrate and the buffer coat materials, respectively[7,8]. Accordingly, even if the CTE mismatch (difference between $\alpha_{\mathrm{s}}$ and $\left.\alpha_{\text {buff }}\right)$ was not practically null or very low, the total thermal stress could be erased when some PBO systems with a considerably low modulus were chosen as the buffer coat materials.

An effect of copolymerization with Si-DA on the tensile modulus $(E)$ is shown in Table 1 and Fig. 3.

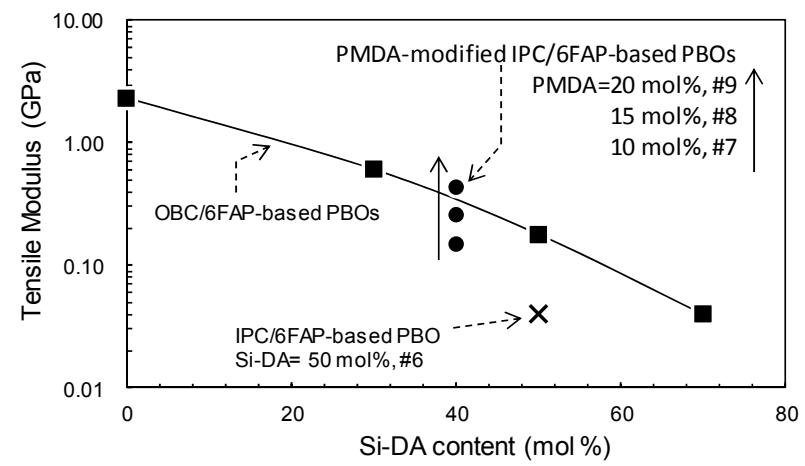

Fig. 3 Effect of Si-DA content on tensile modulus.
The use of Si-DA as a comonomer caused a drastic decrease in the modulus. It should be noted that the PBO gives rise to an extremely low modulus of $0.18 \mathrm{GPa}$ at a Si-DA content of $50 \mathrm{~mol} \%$. Another feature for this system was also observed: significantly enhanced toughness. The PBO film (\#4) showed not only an extremely low modulus of $0.04 \mathrm{GPa}$ but also a drastically enhanced toughness $\left(\varepsilon_{\mathrm{b}}=282 \%\right)$ in spite of a very low molecular weight suggested from its $\eta_{\text {red }}$ value $\left(0.10 \mathrm{dL} \mathrm{g}^{-1}\right)$. The latter is quite different from a general trend for most of polymeric systems; they lose film-forming ability at $\eta_{\text {red }}<\sim 0.3 \mathrm{dL} \mathrm{g}^{-1}$. The pronounced toughening effect for the present systems proves how the siloxane block plays a great role for effective chain entanglement. However, this approach brought about another serious problem: the absence of TMAH solubility at the PHA stage on the basis of strong hydrophobicity of the siloxane block. Actually, the development process using TMAH was disturbed even at a minor siloxane content of $30 \mathrm{~mol} \%$.

\subsection{Siloxane-containing IPC/6FAP-based PBO systems}

The use of IPC $\left(F_{\mathrm{w}}=203.02 \mathrm{~g} \mathrm{~mol}^{-1}\right)$ instead of OBC $\left(F_{\mathrm{w}}=295.12 \mathrm{~g} \mathrm{~mol}^{-1}\right)$ relatively increases in the content of the phenolic $\mathrm{OH}$ groups, as a result, the corresponding siloxane-containing PHAs are expected to show improved TMAH solubility.

Fig. 4 shows FT-IR spectra of a siloxane-containing IPC/6FAP-based thin film (\#6). A strong amide $\mathrm{C}=\mathrm{O}$ stretching band observed at $1645 \mathrm{~cm}^{-1}$ for the PHA film. This band gradually decreased with increasing cure temperature. A careful observation of the spectra shows that this band still remains even after heating at $350{ }^{\circ} \mathrm{C}$. The survived amide group is probably assigned to the amide bond formed between Si-DA and diacid chlrodes; i.e., (-Si-DA-NHCO-DC-) as shown in Fig. 1. Thus, it can be assumed that the PHA is completely converted to PBO upon heating at $350{ }^{\circ} \mathrm{C}$. The poor solubility of the PBOs (e.g., in dimethyl sulfoxide) disturbed the monitoring of cyclodehydration by ${ }^{1} \mathrm{H}-\mathrm{NMR}$ spectroscopy.

Table 1 Film properties of siloxane-containing OBC/6FAP-based PBO systems.

\begin{tabular}{|c|c|c|c|c|c|c|c|}
\hline \multirow[b]{2}{*}{ Entry } & \multicolumn{2}{|c|}{ Monomer } & \multicolumn{2}{|c|}{ PHA } & \multicolumn{3}{|c|}{ PBO } \\
\hline & $\begin{array}{c}6 \text { FAP } \\
(\mathrm{mol} \%)\end{array}$ & $\begin{array}{l}\text { Si-DA } \\
(\mathrm{mol} \%)\end{array}$ & $\begin{array}{c}\eta_{\text {red }} \\
\left(\mathrm{dL} \mathrm{g}^{-1}\right)\end{array}$ & $\begin{array}{l}T_{365} \\
(\%)\end{array}$ & $\begin{array}{c}E \\
(\mathrm{GPa})\end{array}$ & $\begin{array}{c}\varepsilon_{\mathrm{b}} \\
(\%)\end{array}$ & $\begin{array}{c}T_{\mathrm{d}}^{5}\left(\text { in } \mathrm{N}_{2}\right) \\
\left({ }^{\circ} \mathrm{C}\right)\end{array}$ \\
\hline 1 & 100 & 0 & 0.37 & 58.1 & 2.34 & 14 & 532 \\
\hline 2 & 70 & 30 & 0.14 & 42.9 & 0.61 & 64 & 416 \\
\hline 3 & 50 & 50 & 0.25 & 55.3 & 0.18 & 64 & 409 \\
\hline 4 & 30 & 70 & 0.10 & 63.1 & 0.04 & 282 & 419 \\
\hline
\end{tabular}


Actually, the heating condition at $350{ }^{\circ} \mathrm{C}$ seems to be too severe for our siloxane-containing PBO systems because the temperature is higher than the $T_{\mathrm{d}}^{5}$ value in $\mathrm{N}_{2}$ at $331{ }^{\circ} \mathrm{C}$ as listed in Table 2.

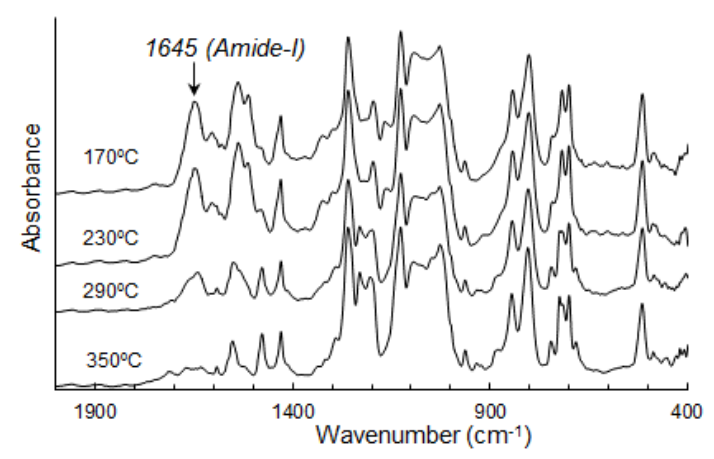

Fig. 4 FT-IR spectra of siloxane-containing IPC/6FAP-based PHA film (\#6) as a function of cure temperature.

Therefore, the all PBO films were prepared upon heating at $300{ }^{\circ} \mathrm{C}$ for $1 \mathrm{~h}$ in vacuum to avoid thermal decomposition for some film property evaluation. The properties of the IPC/6FAP-based siloxane-containing PBO films are summarized in Table 2. The PBO film (\#6) achieved a considerably low modulus of $0.04 \mathrm{GPa}$. For a comparison, this datum was plotted in Fig. 3 [see $(\times)$ ]; the IPC/6FAP-based PBO provided a much lower modulus than the OBC/6FAP-based counterpart when compared at a fixed Si-DA content of $50 \mathrm{~mol} \%$. This is probably attributed to a relatively increased siloxane content in the copolymer (wt $\%$ ) by using a lower molecular weight of IPC than OBC. Unfortunately, all the IPC/6FAP-based siloxane-containing PHAs were essentially insoluble in TMAH against our initial expectation.

\subsection{Siloxane-containing IPC/6FAP-based PBOs} modified with PMDA

In order to improve the TMAH solubility of the PHA without sacrificing the ultra-low-modulus characteristics, a minor fraction of PMDA was copolymerized, by which the carboxylic acid groups with higher acidity (alkali-solubility) than the phenolic $\mathrm{OH}$ groups can be introduced into the siloxane-containing PHAs. Table 3 lists the film properties of the siloxane-containing IPC/6FAP-based systems modified with PMDA (the diamine composition in the copolymer: 6FAP $=60 \mathrm{~mol} \%$, Si-DA $=40 \mathrm{~mol} \%$ ). As expected, this approach drastically improved the TMAH solubility even at minor content of PMDA (10-20 mol\%). Fig. 5 shows FT-IR spectra of the PMDA-modified system (\#9). An increase in cure temperature causes a decrease in the amide $\mathrm{C}=\mathrm{O}$ stretching band at $1656 \mathrm{~cm}^{-1}$ with growth of the imide $\mathrm{C}=\mathrm{O}$ stretching band at $1772 \mathrm{~cm}^{-1}$. A careful observation of the spectra indicates that thermal imidization is completed at a much lower temperature than the oxazole ring formation.

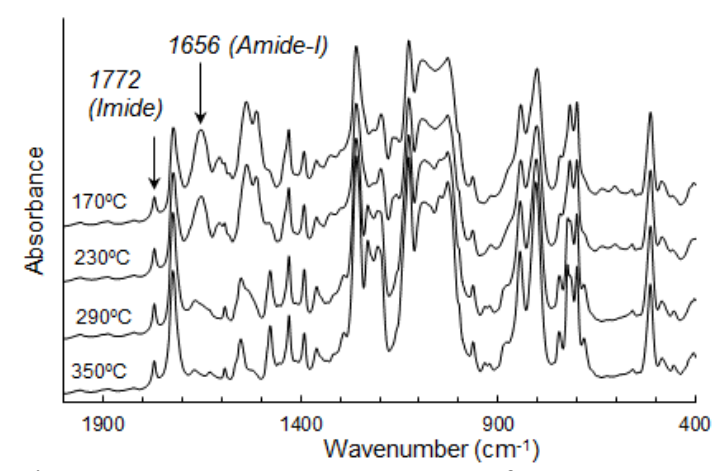

Fig. 5 FT-IR spectra of PMDA-modified IPC/6FAP-based siloxane-containing system (\#9) as a function of cure temperature.

Fig. 6 shows the DMA curves of the siloxane-containing PBO film (\#9). The PBO film possessed a low storage modulus around room temperature, corresponding to the low tensile modulus of $0.44 \mathrm{GPa}$. Another feature was also observed in the DMA curve; the storage modulus more than about $0.07 \mathrm{GPa}$ is maintained until the rubbery specimen turns into a molten state above $135^{\circ} \mathrm{C}$. In contrast, such feature is not observed in another ultra-low-modulus PI system containing butylene oxide block. For a comparison, the tensile modulus of the PMDA-modified systems (\#7-\#9) was plotted in Fig. 3 [see $(\bullet)$ ]. Consequently, the

Table 2 Film properties of siloxane-containing IPC/6FAP-based PBO systems.

\begin{tabular}{|c|c|c|c|c|c|c|c|}
\hline \multirow[b]{2}{*}{ Entry } & \multicolumn{2}{|c|}{ Monomer } & \multicolumn{2}{|c|}{ PHA } & \multicolumn{3}{|c|}{ PBO } \\
\hline & $\begin{array}{l}\text { 6FAP } \\
(\mathrm{mol} \%)\end{array}$ & $\begin{array}{c}\text { Si-DA } \\
(\mathrm{mol} \%)\end{array}$ & $\begin{array}{r}\eta_{\mathrm{red}} \\
\left(\mathrm{dL} \mathrm{g}^{-1}\right)\end{array}$ & $\begin{array}{l}T_{365} \\
(\%)\end{array}$ & $\begin{array}{c}E \\
(\mathrm{GPa}) \\
\end{array}$ & $\begin{array}{l}\varepsilon_{\mathrm{b}} \\
(\%) \\
\end{array}$ & $\begin{array}{c}T_{\mathrm{d}}^{5}\left(\text { in } \mathrm{N}_{2}\right) \\
\left({ }^{\circ} \mathrm{C}\right)\end{array}$ \\
\hline 5 & 60 & 40 & 0.09 & --- & 0.31 & 23 & 377 \\
\hline 6 & 50 & 50 & 0.10 & 51.7 & 0.04 & 36 & 331 \\
\hline
\end{tabular}


minor fractions of the pyromellitimide unit introduced by using PMDA did not unexpectedly contribute to a significant enhancement in the modulus.

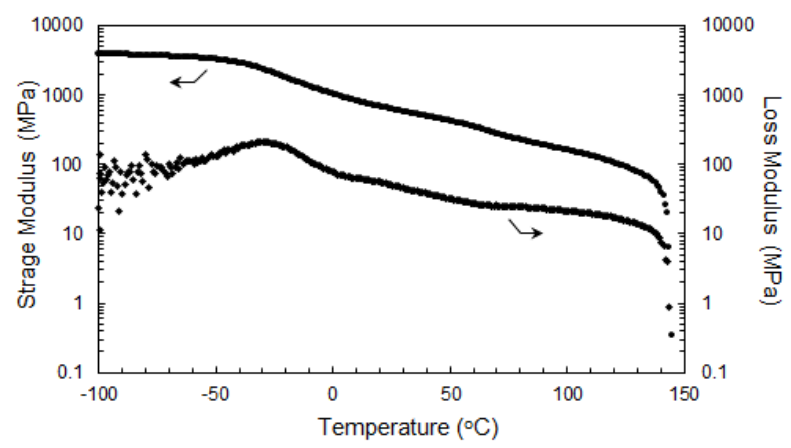

Fig. 6 DMA curves of PMDA-modified IPC/6FAP-based siloxane-containing PBO film (\#9).

\subsection{Photosensitivity of PMDA-modified} IPC/6FAP-based siloxane-containing PHA film

In DNQ-dispersed PHA films, high light transparency of the PHA films themselves at an exposure wavelength (often $i$-line of high-pressure mercury lamp, $365 \mathrm{~nm}$ ) should be maintained from the viewpoints of obtaining higher sensitivity. The 8-10 $\mu \mathrm{m}$-thick DNQ-free PMDA-modified IPC/6FAP-based siloxane-containing PHA films (\#7-\#9) showed a relatively high transmittance transparence higher than $44 \%$ at $365 \mathrm{~nm}$ as listed in Table 3. The results are probably attributed to suppressed charge-transfer (CT) interactions by using an aliphatic diamine, Si-DA and an electron-withdrawing $\mathrm{CF}_{3}$-containing diamine, 6FAP. A previous paper revealed that there exists CT interactions not only in aromatic polyimides but also aromatic polyamides [9]. The present PHAs are the case. Fig. 7 displays a transmission spectrum of the PHA film (\#9) and an absorption spectrum of a dilute GBL solution of DNQ. The spectral comparison indicates that the irradiated light at $365 \mathrm{~nm}$ can be effectively absorbed by the DNQ dispersed in PHA films without an overwhelming shield effect by the matrix polymers. It is known well that unexposed DNQ in a PHA film acts as a dissolution inhibitor against an alkaline solution because of its originally hydrophobic structure. On the other hand, the exposed portion of DNQ is converted to ketene via the Wolff rearrangement, then finally to alkali-soluble indencarboxylic acid in the presence of water as depicted in Fig. 8.

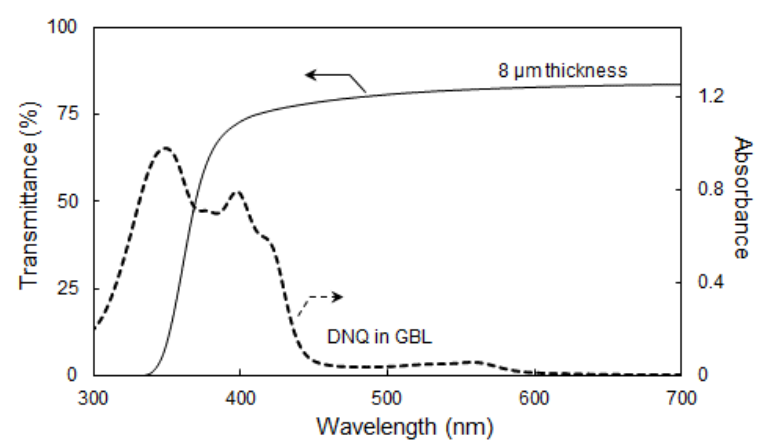

Fig. 7 Transmission spectrum of PHA film (\#9) and UV-visible absorption spectrum of DNQ in GBL.

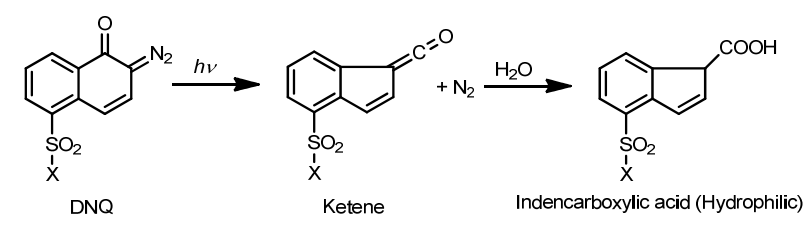

Fig. 8 Photochemical reaction scheme of DNQ.

We first attempted to form a positive-tone pattern from DNQ-containing PHA films. However, insufficient adhesion strength with silicon wafers disturbed fine pattern formation owing to partial removal of the unexposed area during the development process. A combination of adequate silane coupling agents for modifying the PHA solutions and the silicon wafer surface significantly improved the adhesion strength, consequently allowed the formation of positive-tone patterns. Fig. 9 displays a tentatively formed positive-tone pattern with a resolution (line and space) of $40 \mu \mathrm{m}$ as obtained by development using a $2.38 \% \mathrm{TMAH}$ solution at room temperature for $60 \mathrm{sec}$.

Table 3 Film properties of PMDA-modified IPC/6FAP-based PBOs (6FAP 60 mol\%; Si-DA 40 mol\%) systems.

\begin{tabular}{|c|c|c|c|c|c|c|c|}
\hline \multirow[b]{2}{*}{ Entry } & \multicolumn{2}{|c|}{ Monomer } & \multicolumn{2}{|c|}{ PHA } & \multicolumn{3}{|c|}{$\mathrm{PBO}$} \\
\hline & $\begin{array}{l}\text { IPC } \\
(\mathrm{mol} \%)\end{array}$ & $\begin{array}{l}\text { PMDA } \\
(\mathrm{mol} \%)\end{array}$ & $\begin{array}{c}\eta_{\mathrm{red}} \\
\left(\mathrm{dL} \mathrm{g}^{-1}\right)\end{array}$ & $\begin{array}{l}T_{365} \\
(\%)\end{array}$ & $\begin{array}{c}E \\
(\mathrm{GPa}) \\
\end{array}$ & $\begin{array}{c}\varepsilon_{\mathrm{b}} \\
(\%)\end{array}$ & $\begin{array}{c}T_{\mathrm{d}}^{5}\left(\text { in } \mathrm{N}_{2}\right) \\
\left({ }^{\circ} \mathrm{C}\right)\end{array}$ \\
\hline 7 & 90 & 10 & 0.36 & 46.0 & 0.15 & 11 & 399 \\
\hline 8 & 85 & 15 & 0.11 & 44.0 & 0.26 & 9 & 364 \\
\hline 9 & 80 & 20 & 0.11 & 44.8 & 0.44 & 9 & 394 \\
\hline
\end{tabular}




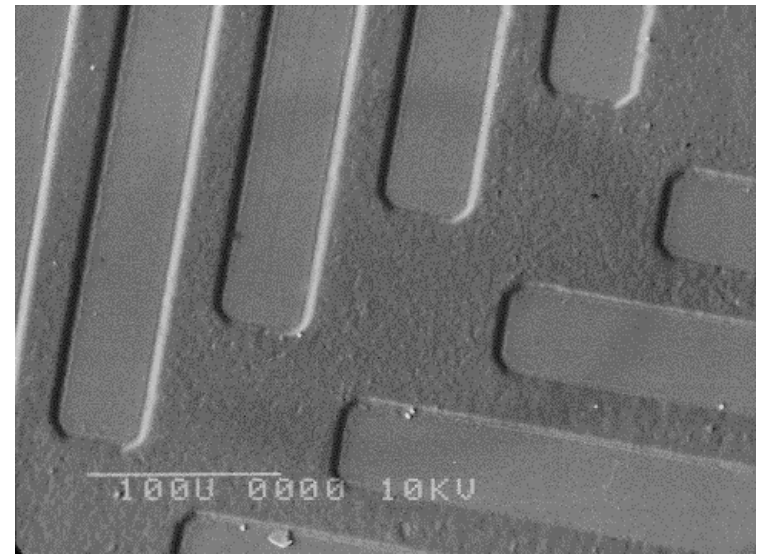

Fig. 9 SEM photograph of positive-tone pattern cured film $(6 \mu \mathrm{m}$ thick $)$ obtained from PMDA-modified IPC/6FAP-based siloxane-containing PHA film (\#9).

\section{Conclusion}

In this work, we obtained novel siloxane-containing PBO precursors with sufficient $i$-line transparency and an adequate TMAH solubility by modifying a minor fraction of PMDA. The DNQ/PHA films allowed to form fine positive-tone patterns by development with $2.38 \%$ TMAH at room temperature.
The corresponding thermally cured PBO films (DNQ-free) achieved a relatively low modulus of $0.44 \mathrm{GPa}$, sufficient thermal stability $\left(T_{\mathrm{d}}{ }^{5}=\right.$ $394{ }^{\circ} \mathrm{C}$ ). Thus, our ultra-low-modulus PBO systems can be promising candidates as novel buffer-coating materials.

\section{References}

1. T. Bamba, Chemical Engineering of Japan, 75, No.2, (2011) 58, in Japanese.

2. M. Hasegawa and A. Ui, J. Photopolym. Sci. Technol., 21, (2008) 151.

3. M. Hasegawa, J. Kobayashi, and L. Vladimirov, J. Photopolym. Sci. Technol., 17, (2004) 253.

4. T. Fukumaru, T. Fujigaya and N. Nakashima, Macromolecules, 45, (2012) 4247.

5. M. Hasegawa, K. Morita, and J. Ishii, J. Photopolym. Sci. Technol., 23, (2010) 495.

6. Y. Maruyama, Y. Oishi, M. Kakimoto and Y. Imai, Macromolecules, 21, (1988) 2305.

7. J.-H Jou and L.-J Chen, Appl. Phys. Lett., 59, (1991) 46.

8. N. Furukawa, Y. Yamada, and Y. Kimura, High Perform. Polym., 8, (1996) 617.

9. M. Hasegawa, Y. Tanaka and A. Tominaga, Polym. Int., 61, (2012) 466. 